\title{
Interannual variability of precipitation distribution patterns in Southern Portugal
}

\author{
S. Mourato, ${ }^{a *}$ M. Moreira ${ }^{b}$ and J. Corte-Real ${ }^{b}$ \\ ${ }^{a}$ Polytechnic Institute of Leiria, Leiria, Portugal \\ ${ }^{\mathrm{b}}$ Institute of Mediterranean Agricultural and Environmental Sciences (ICAAM), Group Water, Soil and Climate, University of Évora, Évora, \\ Portugal
}

\begin{abstract}
Southern Portugal is characterized by an irregular distribution of precipitation which is highly variable from year to year, requiring a clear ascertaining if the pattern of the precipitation is changing due to climate variability or climate change.

In this paper, the authors applied several statistical methods to annual and seasonal time series of precipitation during the period 1931-2006, in order to detect trends and evaluate the variability in the frequencies of occurrence of dry and wet periods and also to assess spatial distribution patterns.

The statistical methods include homogeneity tests, Mann-Kendall test, simple moving averages and cluster analysis. In spring, the authors detected a lack of homogeneity in the precipitation time series and a significant decreasing trend of precipitation was identified, by using simple moving averages and the Mann-Kendall test.

Cluster analyses also confirmed the results and identified significant trends in the interannual and spatial distributions of dry, normal and wet years. It is concluded that the annual precipitation regime in the North interior sector is becoming drier, as the winter and spring also become drier. The only season in which a precipitation increase is observed is at fall and near the sea, but even for that region springs are also becoming drier. Copyright (c) 2009 Royal Meteorological Society
\end{abstract}

KEY WORDS homogeneity; trend analysis; precipitation patterns; clusters techniques; climate variability

Received 14 November 2008; Revised 13 August 2009; Accepted 15 August 2009

\section{Introduction}

Portugal has a mild Mediterranean climate with variability in temporal scales and spatial distribution. One of the main characteristics of the climate in Southern Portugal is the irregular distribution of precipitation and its high interannual variability, not being clearly evident if the pattern of precipitation is really changing in a systematic way. In a climate change scenario it is expected that this region will suffer from higher temperatures, less total annual precipitation and more intense periods of rain all over the year (IPCC, 2007). The purpose of this paper is to seek for some clues in the historical records indicating that the climate in Southern Portugal is already showing trends towards the projected climate change scenarios.

According to the World Meteorological Organization (WMO, 1983), applied climatology aims to make the maximum use of meteorological knowledge, information and consultation for solving social, economic and environmental problems in a practical way. Further, an assessment of the impact of climate variability on human activities must be a major requirement for economic development, social programs and resource management. This issue becomes even more important if we bear in

* Correspondence to: S. Mourato, Polytechnic Institute of Leiria, Leiria, Portugal. E-mail: smourato@estg.ipleiria.pt mind that climate change may have important impacts in future human activities, implying the need for adaptation and mitigation measures.

In the most general sense, the term climatic change includes all forms of climatic inconstancy, regardless of their statistical nature or physical causes. It is often used to designate a change to another climatic state characterized by different averages of atmospheric variables. If a climatic change is characterized by a smooth, monotonic increase or decrease of average values in the period of observation, a climatic trend occurs (WMO, 1983).

In the last $10-15$ years, many studies about climate variability and precipitation changes over the Iberian Peninsula have been published (Rodríguez-Puebla et al., 1998, 2001; Santos et al., 2005; Saenz et al., 2001; Rodrigo and Trigo, 2007; Romero et al., 1998; Zhang et al., 1997), which attested the downward trends in the annual precipitation over Portugal which are more accentuated in the early spring months (Mendes, 1993; Matos et al., 1994; Trigo and DaCamara, 2000; Paredes et al., 2006).

Studies like the one from Santos and Leite (2008) detected increasing trends in maximum temperature for Lisbon mainly during March, and the results from Costa et al. (2008) about the topographic characteristics and spatial climatic diversity indicate that extreme precipitation has decreased through time in Southern Portugal. 